\title{
Relationship among plasminogen activator inhibitor-1, bone mineral density, metabolic and bone turnover markers in postmenopausal women with type 2 diabetes mellitus
}

S. Canecki-Varžić, I. Prpić-Križevac, I. Bilić-Ćurčić

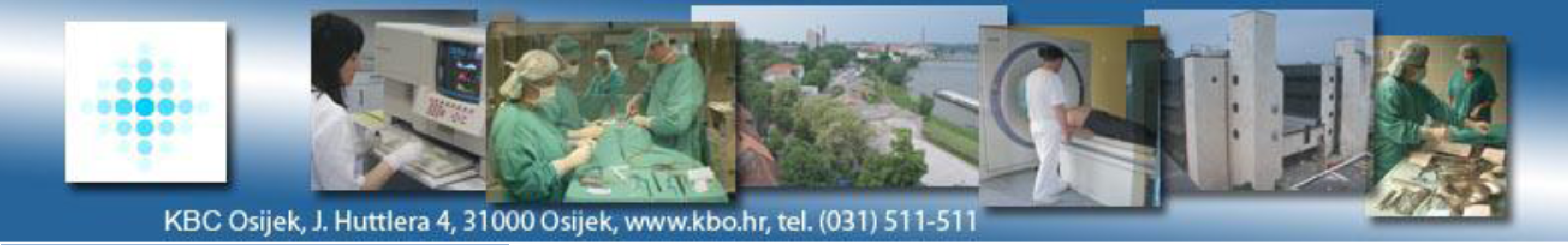

\section{Clinical Hospital Center Osijek}

\section{BACKGROUND}

Women with type 2 diabetes mellitus (T2DM) have a higher risk of fractures despite increased bone mineral density (BMD). In experimental studies a potential role of plasminogen activator inhibitor-1 (PAl-1) in bone remodeling is suggested but studies in humans are lacking.

\section{OBJECTIVE}

This is a first study in humans investigating whether circulated levels of PAI-1 in postmenopausal women with T2DM are related to BMD and adiposity.

\section{RESULTS}

Diabetic patients with normal BMD had significantly higher BMI, greater waist circumference and lower bone turnover markers than diabetics with osteopenia and osteoporosis $(p<0,01)$. PAl-1 was lower in diabetics with osteoporosis and osteopenia compared with diabetics with normal BMD $(p<0,05)$. In the multiple regression models the strongest determinants of PAI-1 among metabolic parameters were triglyceride and insulin levels and the duration of T2DM, among therapy beta blockers, and among bone markers pyrilinks $(p<0,05)$. Final regression analysis model revealed insulin $(p=0.003)$, triglycerides levels $(p=0.0002)$ and pyrilinks $(p=0.0002)$ to be the strongest predictors of PAI-1 levels in all patients.

Associations of PAI-1 levels with metabolic parameters, BMD and bone turnover markers

\begin{tabular}{lcc}
\hline \multicolumn{1}{c}{ PAl-1 } & $\mathrm{r}$ & $\mathrm{p}$ \\
\hline Age (year) & $-0,2375$ & 0,011 \\
BMI $\left(\mathrm{kg} / \mathrm{m}^{2}\right)$ &, 3453 & 0,000 \\
Diabetes duration (year) &,- 3046 & 0,002 \\
Insulin $(\mathrm{mlU} / \mathrm{L})$ &, 4411 & 0,000 \\
Triglycerides $(\mathrm{mmol} / \mathrm{L})$ &, 4704 & 0,000 \\
HDL $(\mathrm{mmol} / \mathrm{L})$ &,- 3331 & 0,001 \\
Osteocalcin $(\mathrm{ng} / \mathrm{ml})$ &,- 2976 & 0,002 \\
Pyrilinks $(\mathrm{nMPD} / \mathrm{mMc})$ &,- 2897 & 0,002 \\
Crosslaps $(\mathrm{ng} / \mathrm{ml})$ &,- 2955 & 0,002 \\
Lumbar BMD $\left(\mathrm{g} / \mathrm{cm}^{2}\right)$ &, 1990 & 0,046 \\
Hip BMD $\left(\mathrm{g} / \mathrm{cm}^{2}\right)$ &, 3530 & 0,000 \\
\hline
\end{tabular}

\section{METHODS}

Anthropometric variables, PAI-1 and insulin levels, serum lipids and bone turnover markers were measured in 117 postmenopausal women with T2DM. A total of 117 female patients were divided according to lumbar spine BMD measurements via dual-energy $\mathrm{x}$-ray absorptiometry in three groups: 47 with osteopenia, 21 with osteoporosis and 49 with normal BMD.

Table 1. Baseline characteristics of study subjects

\begin{tabular}{|c|c|c|c|c|c|c|c|}
\hline \multirow[b]{2}{*}{ Age (year) } & \multicolumn{2}{|c|}{ Osteoporosis $n=21$} & \multicolumn{2}{|c|}{ Osteopenia $n=47$} & \multicolumn{2}{|c|}{$\begin{array}{c}\text { Normal BMD } \\
n=49\end{array}$} & \multirow{2}{*}{$\begin{array}{c}\mathrm{p} \\
\mathrm{NS}\end{array}$} \\
\hline & 65,3 & 10,2 & 67,4 & 8,04 & 64,9 & 8,7 & \\
\hline Waist (cm) & 89,8 & 11,2 & 96,0 & 8,2 & 98,3 & 12,0 & $\ddagger 0,03 ; \uparrow 0,003$ \\
\hline BMI $\left(\mathrm{kg} / \mathrm{m}^{2}\right)$ & 27,5 & 5,6 & 29,1 & 3,9 & 31,3 & 5,1 & ${ }^{*} 0,02 ; \uparrow 0,002$ \\
\hline Menopause duration (year) & 17,8 & 9,2 & 18,3 & 8,5 & 14,7 & 7,9 & ${ }^{*} 0,049$ \\
\hline Diabetes duration (year) & 9,1 & 6,3 & 12,2 & 7,1 & 12,1 & 7,3 & NS \\
\hline HbA1c (\%) & 10,2 & 2,24 & 10,1 & 1,59 & 9,95 & 1,9 & NS \\
\hline Cholesterol (mmol/l) & 5,45 & 1,62 & 5,13 & 1,25 & 4,97 & 1,41 & NS \\
\hline HDL cholesterol (mmol/l) & 1,29 & 0,25 & 1,22 & 0,25 & 1,19 & 0,27 & NS \\
\hline LDL cholesterol (mmol/l) & 3,39 & 1,02 & 3,03 & 1,02 & 2,76 & 1,12 & ${ }^{*} \dagger 0,042$ \\
\hline Triglicerydes (mmol/l) & 1,98 & 1,32 & 1,96 & 0,79 & 2,37 & 1,43 & NS \\
\hline Osteocalcin (ng/ml) & 10,22 & 7,67 & 8,37 & 3,84 & 6,32 & 2,87 & ${ }^{*} 0,025 ; † 0,0013$ \\
\hline Insulin (mIU/l) & 8,94 & 4,14 & 9,97 & 4,82 & 10,62 & 5,69 & NS \\
\hline Pyrilinks (nMPD/mMc) & 7,8 & 3,1 & 7,41 & 2,9 & 5,5 & 2,1 & ${ }^{*} 0,001 ; \uparrow 0,0015$ \\
\hline Crosslaps (ng/ml) & 0,54 & 0,25 & 0,49 & 0,29 & 0,35 & 0,21 & ${ }^{*} 0,012 ; \dagger 0,0092$ \\
\hline PAI-1 (U/l) & 3,3 & 2,1 & 3,5 & 2,1 & 4,4 & 1,9 & ${ }^{*} 0,042 ; \uparrow 0,052$ \\
\hline Fibrinogen (g/L) & 4,1 & 1,0 & 4,3 & 0,8 & 4,0 & 0,8 & NS \\
\hline $\mathrm{CRP}(\mathrm{mg} / \mathrm{L})$ & 5,77 & 11,6 & 3,89 & 3,67 & 3,74 & 4,25 & NS \\
\hline Lumbar BMD (g/cm²) & 0,707 & 0,07 & 0,856 & 0,04 & 1,036 & 0,09 & *† $\ddagger<0,05$ \\
\hline Hip BMD $\left(\mathrm{g} / \mathrm{cm}^{2}\right)$ & 0,746 & 0,13 & 0,838 & 0,109 & 0,985 & 0,147 & ${ }^{*} \ddagger ; \dagger<0,05$ \\
\hline
\end{tabular}

Final stepward forward regression analysis model with therapy (including age, BMI, diabetes duration, menopause duration, insulin, triglycerides, femoral and lumbar BMD)

\begin{tabular}{lrcc}
\hline Dependent variable: PAI-1 & \multicolumn{1}{c}{ Beta } & adjusted $\mathrm{R}^{2}$ \\
\hline & & & \\
Triglycerides (mmol/L) & 0.314 & 0.0002 & 0.574 \\
Insulin (mIU/L) & 0.250 & 0.0033 & \\
Pyrilinks (nMPD/mMc) & -0.308 & 0.0002 & \\
BMI (kg/m²) & 0.310 & 0.0035 & \\
Waist circumference (cm) & -0.278 & 0.0097 & \\
Age (years) & -0.364 & 0.0201 & \\
Diabetes duration (years) & -0.187 & 0.0185 & \\
Menopause duration (years) & 0.283 & 0.0688 & \\
Beta blockers (\%) & 0.224 & 0.0045 & \\
\hline
\end{tabular}

\section{CONCLUSION}

Conclusions: Our findings suggest that the PAI-1 has a protective effect on bone loss by suppression of bone turnover in obese diabetic patients, and the effect is primarily mediated through the influence of metabolic factors, hyperinsulinemia, hypertriglyceridemia and obesity. However, the fact that pyrilinks is also independently correlated to PAI-1 implies its direct involvement in bone metabolism influencing bone mass and strength. 